P6 (continued)

(FMI) integrated nutrition education program among 4 th grade students, as compared to a similar group of control students.

Study Design, Settings, Participants, Intervention: The sample consisted of $n=263$ students nested in 18 classrooms in North Carolina and Ohio. Nine classrooms used FMI and nine used traditional mathematics and science curriculum in a quasi-experimental design.

Outcome Measures and Analysis: Multi-level models were used to assess the relationship between student-level variables including: gender, free and reduced lunch status (as a proxy for student socioeconomic status), and baseline nutrition knowledge; classroom-level variables including: control or intervention status, teacher experience and teacher nutrition training on student nutrition knowledge at the conclusion of the program. A total of five models were built and analyzed using PROC MIXED and estimated using maximum likelihood in SASv9.4.

Conclusions and Implications: Classroom-level variables including curriculum and teacher characteristics accounted for $\sim 56 \%$ of variability in nutrition knowledge after the program. The FoodMASTER intervention was found to be a significant predictor of nutrition knowledge. Students exposed to FMI score on average 4.32 points higher than control classrooms $(\mathrm{p}=.0001)$. Teachers with $8+$ years of experience were found to improve predicted post-nutrition knowledge scores $(\mathrm{p}=.006)$. Based on these analyses, integrated nutrition education such as FoodMASTER significantly increases student nutrition knowledge. Further, there is a moderating influence of teacher experience indicating that effectiveness of nutrition education programs may be improved when administered by experienced teachers.

Funding: None

\section{P7 Clustering of Youth Empowerment and Health Behaviors Among Sixth to Eighth Grade Adolescents}

Biyi Chen, MS, South Dakota State University; Kendra Kattelmann, PhD, RDN, LN, FAND, kendra. kattelmann@sdstate.edu, South Dakota State University, Box 2203, Health and Nutritional Sciences Department, Brookings, SD 57006; Ann Michelle Daniels, PhD, South Dakota State University; Koushik Adhikari, PhD, University of Georgia; Susan Zies, MEd, Ohio State University Extension; Erika Lindshield, MPH, RDN, $L D$, Kansas State University; Tandalayo Kidd, $P h D, R D, L P N$

Objective: To identify clusters of perceived youth empowerment among sixth to eighth grade adolescents and their cross-sectional associations with demographic indicators; to examine the distribution of health behaviors (including nutrition and physical activity (PA) behaviors), as identified clusters, among genders.

Study Design, Settings, Participants, Intervention: This baseline study was conducted as part of a five-year tri-state community-based participatory research (CBPR) project-Ignite: Sparking Youth to Create Healthy Commu- nities. A total of 282 six to eighth grade adolescents from South Dakota, Kansas and Ohio completed questionnaires related to youth health behaviors and perceived youth empowerment.

Outcome Measures and Analysis: The cluster analysis identified groups of adolescents with similar perceived youth empowerment. Chi-square tests assessed cluster differences by gender, ethnicity, and urban/rural status. Analyses were conducted using SAS 9.4 software.

Results: Two clusters were identified empowered youth (EY) $(n=90)$ and "un-empowered youth (UY)" $(\mathrm{n}=192)$. For both genders, the "EY" cluster had significantly greater ( $\mathrm{P}$ $<0.05$ ) health promoting behaviors which included greater self-efficacy, motivation, perceived social support in regards to healthy behaviors, positive health beliefs and less perceived barriers to healthy eating and PA when compared to UY. In addition, the youth in the EY cluster reported more time for PA (total at least 60 minutes/day) per week.

Conclusions and Implications: Promoting youth empowerment may be an effective way of informing interventions designed to foster engagement in health-promoting behaviors among adolescents. Further research should investigate the influence for youth empowerment to promote the health development in youth and create greater community change.

Funding: NIFA

\section{P8 Cognitive Interviews Define Fuel for Fun Physical Activity Survey Items as Face Valid with Rochester, NY Area Fourth Graders}

Elizabeth Wayman, MS, Rochester Institute of Technology; Leslie Cunningham-Sabo, PhD, RD, Colorado State University; Barbara Lohse, PhD, RD, CDN, balihst@rit.edu, Rochester Institute of Technology, 180 Lomb Memorial Drive, CIMS 78-A622, Rochester, NY 14623

Objective: Assess face validity of tested physical activity (PA) self-report items used to evaluate Fuel for Fun with a new 4th grade audience.

Study Design, Setting, Participants: Face-to-face, audio-recorded cognitive interviews conducted at library or school venues with a convenience sample of children from the Rochester, NY area.

Outcome Measures and Analysis: Interview transcripts were analyzed with Atlas.ti using a content analysis approach. Coherence and congruence with reference definitions were assessed for descriptions or amount of strenuous, moderate, mild, sedentary activity and practices/intentions toward PA to denote stage of change for PA behavior.

Results: Students ( $\mathrm{n}=24 ; 50 \%$ girls; $92 \%$ white; $12 \%$ Hispanic) ranged from 8 to $10 \mathrm{y}(\mathrm{M}=9.70 \pm .43 \mathrm{y})$ and attended nine schools (three urban, six suburban). Four students attended schools with free/reduced lunch participation $\geq 50 \%$ of the student body. Mean interview length was $29 \pm 4 \mathrm{~min}$. Stage of change was action/maintenance for $87 \%$, with $172 \pm 110 \mathrm{~min} /$ week strenuous activity, $155 \pm 122 \mathrm{~min} /$ week moderate, $124 \pm 136 \mathrm{~min} /$ week mild, and $2.5 \pm 2.2$ hours/day sedentary activity. Although only $17 \%$ demonstrated comprehension of the terms strenuous,

Continued on page S26 